\title{
Parity Dependent Shell Model Level Densities for Nuclear Astrophysics
}

\author{
Mike Scott ${ }^{* \dagger}$ \\ Department of Physics, Central Michigan University, Mount Pleasant, Michigan 48859, USA \\ E-mail: Scott1MJ@CMich.edu

\section{Mihai Horoi ${ }^{\ddagger}$} \\ Department of Physics, Central Michigan University, Mount Pleasant, Michigan 48859, USA \\ E-mail: Horoi@phy.cmich.edu
}

\begin{abstract}
Recently, we developed a methodology [1-4] of calculating the spin and parity dependent shell model nuclear level density, which is a very useful ingredient in the Huaser-Feshbach theory for calculating reaction rates for nuclear astrophysics[5]. We developed new techniques based on nuclear statistical spectroscopy [6] to calculate the spin and parity projected moments of the nuclear shell model Hamiltonian, that can be further used to obtain an accurate description of the nuclear level density up to about $15 \mathrm{MeV}$ excitation energy. These techniques were fully tested for the $s d$-shell nuclei and some light $f p$-shell nuclei, by comparing with the level density obtained from exact shell model diagonalization. Here we present for the first time comparisons with the exact shell model diagonalization for nuclei heavier than ${ }^{56} \mathrm{Ni}$, in a model space spanned by the $f_{5 / 2}, p_{3 / 2}, p_{1 / 2}$ and $g_{9 / 2}$ orbits. The ratio of nuclear level densities of opposite parities is also discussed. This analysis was possible due to a new and very efficient nuclear shell model code [7] that can provide a large number of states of given spin and parity.
\end{abstract}

10th Symposium on Nuclei in the Cosmos

July 27 - August 12008

Mackinac Island, Michigan, USA

\footnotetext{
*Speaker.

${ }^{\dagger}$ The authors acknowledge computational support from the NSF MRI Grant No. PHY- 0619407.

¥Acknowledging support from NSF Grant No. PHY-0758099, and the DOE Grant No. DE-FC02-07ER41457.
} 
Spin- and parity-dependent nuclear level densities represent an important ingredient for the Hauser-Feschbach theory of nuclear reactions. In most of the cases relevant to nuclear astrophysics, where experimental information is not available, the reaction rates for medium and heavy nuclei can only be estimated using the Hauser-Feshbach approach. Nuclear level densities are usually obtained using the back-shifted Fermi gas (BSFG) approximation[5], which was improved over the years. More modern approaches to the level densities based on the mean-field were recently proposed by S. Goriely and collaborators[11, 12].

These approximations assume an independent particle model in a mean-field, which lack information about the many body correlations. These correlations can be included exactly if one can fully diagonalize the many body nuclear Hamiltonian, a task of increased difficulty. Alternatively, one can use Monte-Carlo techniques [13], or methods of the statistical spectroscopy[6]. We recently developed a methodology $[1,2,3,4]$ of calculating the spin and parity dependent shell model nuclear level densities, which is a very useful input to the Huaser-Feshbach theory for calculating reaction rates for nuclear astrophysics[5]. We developed new techniques based on nuclear statistical spectroscopy [6] to calculate the configuration spin and parity projected moments of the nuclear shell model Hamiltonian, that can be further used to obtain an accurate description of the nuclear level density up to about $15 \mathrm{MeV}$ excitation energy.

Using the moments method of finding nuclear level densities (NLD), we evaluated parity dependent nuclear level densities for ${ }^{66} \mathrm{Zn}$ and ${ }^{64} \mathrm{Ge}$, for $\mathrm{J}=0-6$ and $\mathrm{J}=0-12$ respectively, in the $f p g$ model space defined by the valence spherical orbits $f_{5 / 2}, p_{3 / 2}, p_{1 / 2}$ and $g_{9 / 2}$. The effective interaction matrix elements were chosen from the G-matrix with core polarization, and were further tuned to describe energy levels of a number of nuclei above the ${ }^{56} \mathrm{Ni}$ core (details will be reported soon). Several studies using pairing plus quadrupole interaction [7] suggest that the $1 d_{5 / 2}$ orbit may be important in this region. We did not include it in this study. The results are accurate well above the energy range of interest for nuclear astrophysics [5] as seen in Figs. 1 and 3. The case of ${ }^{66} \mathrm{Zn}$ was chosen for future comparison to the experimental data [8], while ${ }^{64} \mathrm{Ge}$ was chosen because it is one of the waiting points in the rp-process path[14].

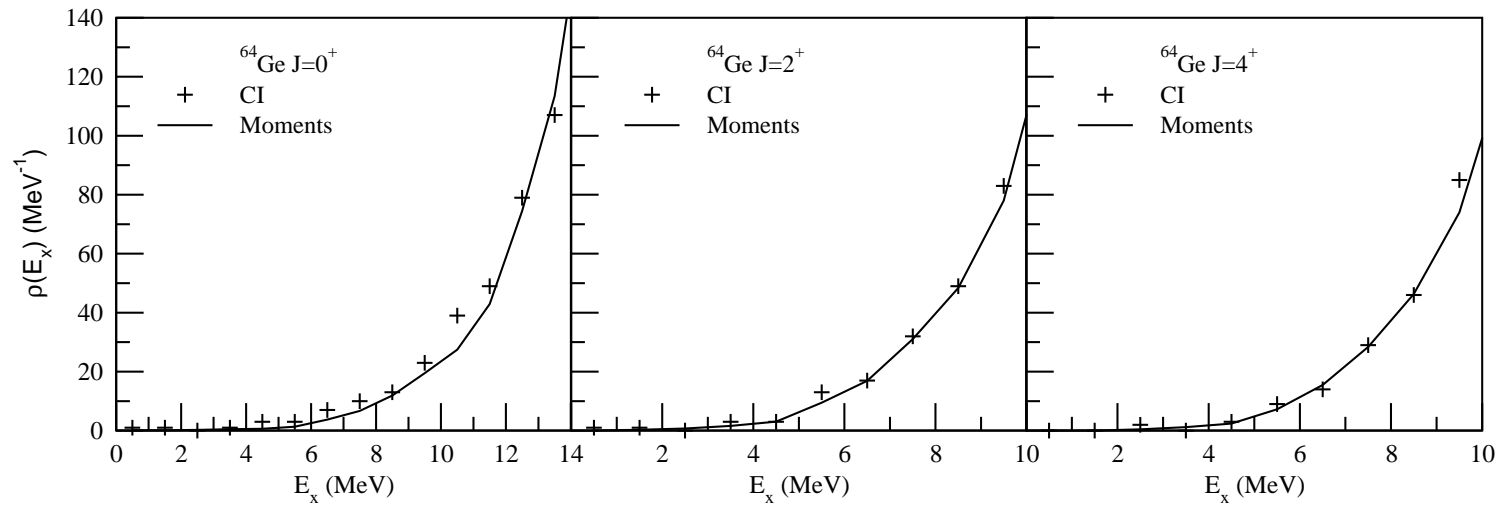

Figure 1: Comparison of moments method NLD (full line) and CI methods NLD (+) for some selected $\mathrm{J}$-values in ${ }^{64} \mathrm{Ge}$ 


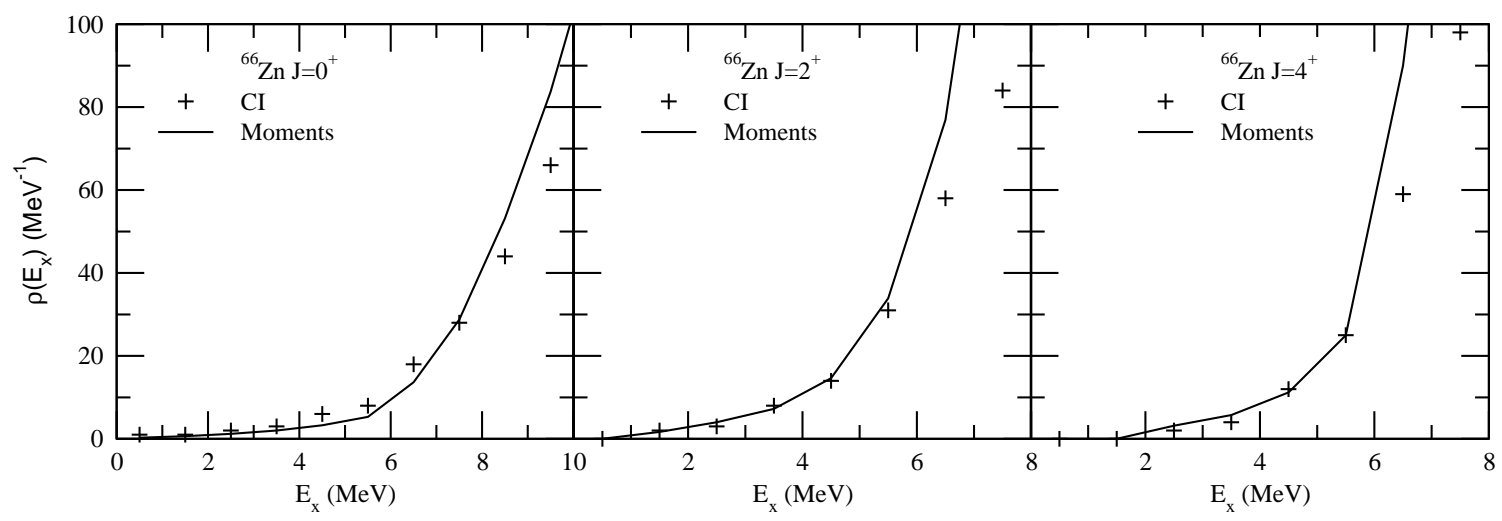

Figure 2: Comparison of moments method NLD (full line) and CI methods NLD (+) for some selected $\mathrm{J}$-values in ${ }^{66} \mathrm{Zn}$.

We also calculated the NLD using the Configuration Interaction (CI) method with the same nuclear Hamiltonian used with the moments method, for which we use the newly developed J-scheme CI code NuShellX [7]. Although this CI code is ideal for calculating nuclear level densities, it is still restrictive in the amount of time it takes for calculation, due to the requirement of calculating hundreds of energies for the same J. For a single J value of one isotope the CI calculation may take days to weeks, depending on complexity of the nucleus and number of levels to be calculated.

Figure 1 shows a comparison between NuShellX's configuration interaction (CI) method of calculating NLD's and the moments method for ${ }^{64} \mathrm{Ge}^{\pi}=0^{+}, 2^{+}$, and $4^{+}$. The moments NLDs compare well with the $\mathrm{CI}$ for the major contributing states with less than $14 \%$ discrepancy. Figure 2 shows the same comparison for ${ }^{6} \mathrm{Zn}{ }^{\pi}=0^{+}, 2^{+}$, and $4^{+}$. In this case the discrepancy is higher with $0^{+}$and $2^{+}$with less than $23 \%$ discrepancy, but the $4^{+}$has $34 \%$ and in all cases can be seen to diverge at higher energy.

As seen in Figs. 1 and 2 the CI NLD could only be calculated up to 8-14 MeV. However, the agreement between the two methods up to that energy suggests the one can extrapolate the moments method results beyond that range. As already mentioned, the moments method uses a sum of finite range Gaussians for the all possible configurations of the valence nucleons in spherical single particle orbits,

$$
\rho\left(E_{x}, J, \pi\right)=\sum_{c \in \text { conf }} D_{c}(J, \pi) G_{F R}\left(E, E_{c}(J), \sigma_{c}(J)\right)
$$

where $D_{c}$ is the dimension of each configuration, $G_{F R}$ is the finite range Gaussian, $E_{c}(\mathrm{~J})$ and $\sigma_{c}(\mathrm{~J})$ are the first two moments for each configuration, $E_{x}$ is the excitation energy, and $\pi$ is the parity. One area requiring further investigation is where for each configuration's finite range Gaussian one should truncate the energy range. As suggested in Ref. [4] we began investigations using (3-4) $\sigma$ and bounded by the yrast energy for each spin in the fpg model space. It became evident that values of (2-4) $\sigma$ better described the CI NLD's, and we have begun an investigation of the magnitude of deviations between the level densities calculated with these two methods using the quantity, 


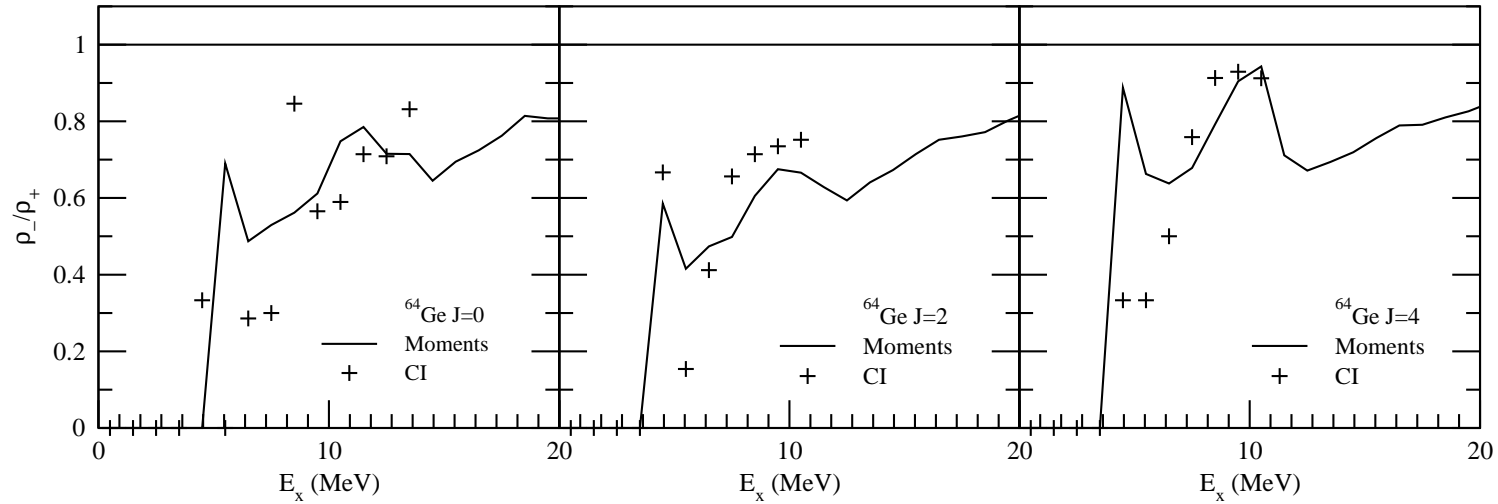

Figure 3: Parity ratio of NLD from moments method (full line) and CI method (+) for ${ }^{64} \mathrm{Ge}$.

$$
\varepsilon=\frac{\sum_{E_{i}}\left|\rho_{C I}\left(E_{i}\right)-\rho_{\text {mom }}\left(E_{i}\right)\right|}{\sum_{E_{i}} \rho_{C I}\left(E_{i}\right)}
$$

as a figure of merit. Here $E_{i}$ are the middle-bin excitation energies taken up to the maximum calculated with the CI method, and the sum is restricted to nonzero CI NLD for the lower end of the spectrum. The complete investigation is still pending, but preliminary results using equation (2) show that one can reduce the deviations between the moments method and the full CI to lower than $10 \%$. We believe that the lower bound of the yrast state energies may be contributing to a skewing of the finite range Gaussians resulting in moments that do not well describe the configuration. We are currently working on a method that will adjust the moments to account for this skewing.

One drawback of BSFG and other mean-field approaches to NLD is that they predict equal contributions to the negative parity NLD as for the positive parity NLD at a given energy. It is well know that this assumption is inaccurate, especially at lower excitations energies of interest in nuclear astrophysics. The CI and the moments methods do not suffer of this drawback if the valence space can describe both parities, as is the case with the fpg valence space described above.

Figs. 3 and 4 show the density ratio of negative and positive parity for ${ }^{64} \mathrm{Ge}$ and ${ }^{64} \mathrm{Zn}$ states corresponding to the same $\mathbf{J}$ values respectively. The major point here is the ratio being less than 1 for low energies less than $10 \mathrm{MeV}$. Convergence to 1 occurs for energies greater than $20 \mathrm{MeV}$ [9]. For ${ }^{64} \mathrm{Ge} \mathrm{J}^{\pi}=2^{+}$and $4^{+}$and ${ }^{66} \mathrm{Zn} \mathrm{J}{ }^{\pi}=0^{+}$and $2^{+}$the threshold energies are accurately described by this method. The states not mentioned may be affected by the aforementioned skewing of the Gaussians and large bin sizes.

The right panel in Fig. 4 shows a CI bin NLD to the left of the moments method NLD threshold. This is due to the larger bin size of $1 \mathrm{MeV}$ used in the calculation associated with a threshold energy that is not on $1 \mathrm{MeV}$ boundary. This problem can be overcome by reducing the size of the energy bins, with little loss in computing time. Several checks have shown this to improve the behavior of the lower energy NLD.

In conclusion we used the spin and parity configurations moments method to calculate NLD for several nuclei of interest for the rp-process, using the fpg valence space that can describe both positive and negative parities for these nuclei. We were able for the first time to compare the 


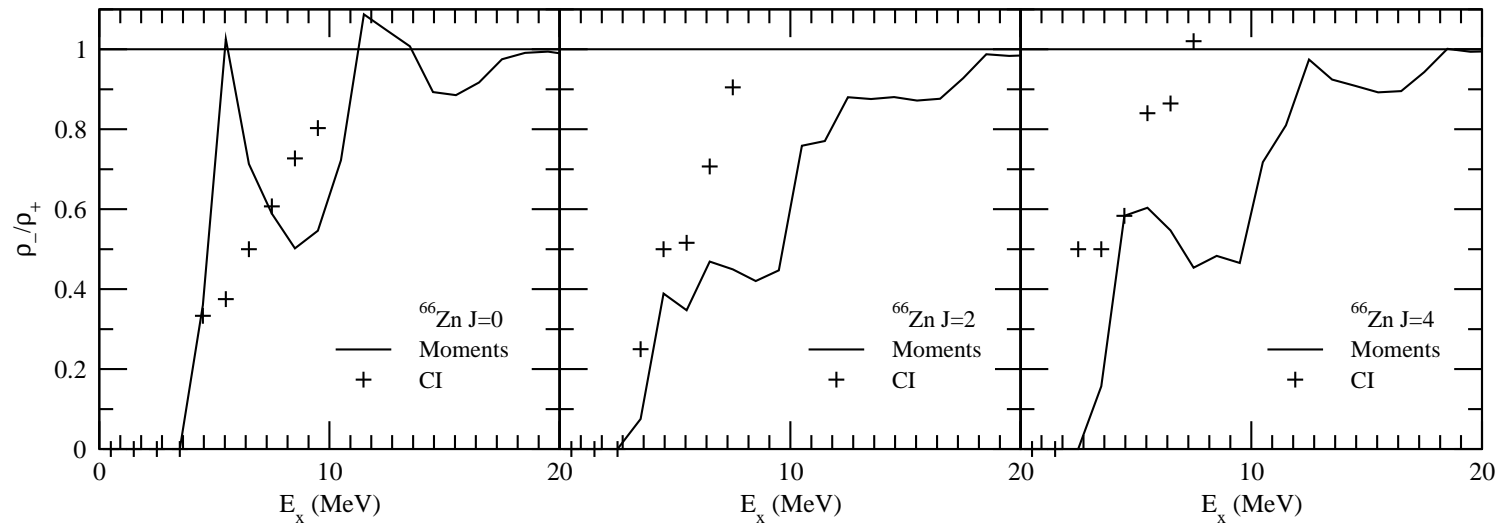

Figure 4: Parity ratio of NLD from moments method (full line) and CI method (+) for ${ }^{66} \mathrm{Zn}$.

approximate results of the moments method with the exact CI calculations in this large model space by taking advantage of the new J-scheme CI code NuShellX. Our calculated level densities show very good agreement between the moments method NLD and those provided by NuShellX. We also calculated the ratio of negative and positive NLD with both methods at low energies of interest for astrophysics, and show that it can be significantly different from 1 . We also found reasonable good agreement between the two methods for this quantity. These findings suggest that one could use the moments method to accurately describe the CI NLD for nuclei and energy ranges that are unreachable to the CI method.

\section{References}

[1] M. Horoi and V. Zelevinsky, Phys. Rev. Lett. 98, 262503 (2007).

[2] M. Horoi, M. Ghita and V. Zelevinsky, Nucl. Phys. A 785C, 142 (2005).

[3] M. Horoi, M. Ghita and V. Zelevinsky, Phys. Rev. C 69, 041307(R) (2004).

[4] M. Horoi, J. Kaiser and V. Zelevinsky, Phys. Rev. C 67, 054309 (2003).

[5] Th. Rauscher, F.-K. Thielemann, and K.-L. Kratz, Phys. Rev. C 56, 1613 (1997).

[6] S.S.M. Wong, Nuclear Statistical Spectroscopy, Oxford, 1986.

[7] K. Langanke et al., Nucl. Phys A 728, 109 (2003); M. Hasegawa et al., Nucl. Phys. A 789, 46 (2007).

[8] W. Rae, NuShellX (2008), http://knollhouse.org/NuShellX.aspx.

[9] National Nuclear Data Center, Brookhaven National Laboratory. (2008, March 18). NuDat (Nuclear Structure and Decay Data). Retrieved August 22, 2008, from http://www.nndc.bnl.gov/nudat2/

[10] M. Horoi, Proc. Sci., (NIC-IX) (2006) 120 [http://pos.sissa.it/].

[11] http://www-astro.ulb.ac.be/Html/nld.html

[12] http://www-astro.ulb.ac.be/Html/nld_comb.html

[13] Y. Alhassid, S. Liu, and H. Nakada, Phys. Rev. Lett. 99, 162504 (2007).

[14] H. Schatz, at al., Phys. Rep. 294, 167 (1998). 